\title{
Primary neurendocrine tumor of the liver, an exceptional location
}

\begin{abstract}
The digestive endocrine tumors have mainly gastrointestinal and duodenopancreatic localization. Liver metastases are frequent there. The primary hepatic localization remains exceptional. We report a new observation revealed in a 42-year-old man with an abdominal pain and deterioration in general condition. The CT scan and histologic appearance of the percutaneous biopsy were in favor of hepatocellular carcinoma. The immunohistochemical study showed the endocrine nature of the tumor. The various endoscopic and morphological examinations, in particular the octreoscan, eliminated any extra-hepatic localization, which made it possible to retain the primary hepatic origin of the tumor.
\end{abstract}

Keywords: endocrine tumor, liver, immunohistochemistry, octreoscan
Volume II Issue 6 - 2020

\author{
Ouaya H,' Benjouad K,' Aiterrami A,' \\ Oubaha S, ${ }^{2}$ Samlani Z,' Bouyali $C,{ }^{3}$ elhadri \\ $\mathrm{K},{ }^{3}$ Boutakioute $\mathrm{B},{ }^{3}$ Ouali Idrissi $\mathrm{M},{ }^{3}$ Cherif \\ guenoun El Idrissi $\mathrm{N}^{3}$ Krati $\mathrm{K}^{\prime}$ \\ 'Department of Gastroenterology, Mohammed VI University \\ Hospital, Morocco \\ ${ }^{2}$ Department of Physiology, Faculty of Medicine at Cadi Ayyad \\ University, Morocco \\ ${ }^{3}$ Department of radiology, Mohammed VI University Hospital, \\ Morocco
}

Correspondence: Hassan Ouaya, Mohammed VI University Hospital, Marrakech, Morocco, Tel +2/2654830906, Email dr.ouaya.h@gmail.com

Received: November 03, 2020 | Published: December 16 2020
Abbreviations: CgA, chromogranin A; 5HIAA, 5-hydroxyindole acetic acid, NETs, Neuroendocrine tumors

\section{Introduction}

Neuroendocrine tumors account for between 1 and $2 \%$ of all tumor pathologies with digestive localization. However, the hepatic localization remains extremely rare, since the first publication by EDMEN$\mathrm{SON}^{1}$ in 1958, until our day, the number of the cases, to publish, not exceeding 150 cases, making intoxicate of $0.3 \%{ }^{2}$ of all neuroendocrine tumors, which makes the management, either diagnostic or therapeutic, not yet codified.

\section{Case report}

A 42years old patient, without a specific pathological history, presented in our institution for with intermittent minimal intensity abdominal pain. There were no other digestive manifestations. He reported a weight loss estimated at $5 \mathrm{~kg}$ in 6 months, with conserved appetite Clinical examination, hepatomegaly, painless, with a bumpy outline with hepatic arrow at $18 \mathrm{~cm}$.

Biologically assessment had shown normocytic normochromic anemia at $9.5 \mathrm{~g} / \mathrm{dl}$, and platelet count $354,000 / \mathrm{mm} 3$, tumor markers CA 19-9 and ACE, $\alpha$-fetoprotein were normal. An abdominal ultrasound completed, by the abdominal CT were done, objectifying: Liver is riddled with nodules, solid, hypodenses, the largest hypovascular. Measuring $42 \mathrm{~mm}$, without dilation of the bile ducts, with slight wall thickening in the gastric antrum, absence peritoneal effusion, with normal looking kidneys and pancreas head. Endoscopic digestive exploration was normal (gastroscopy, colonoscopy). A liver biopsy done, with the histological study: a carcinomatous proliferation, the immunohistochemical complement showed a positivity of the tumor cells for chromogranin, synaptophysin and CK 19. With labeling by Ki67 estimated at 15\%, in favor of a well-differentiated grade 2 neuroendocrine tumor of the WHO 2010 classification of Digestive NETs. Faced with this histological discovery, a biological assessment (deter- mination of serotonin (5HT), 5-hydroxyindole acetic acid (5HIAA), chromogranin $\mathrm{A}(\mathrm{CgA})$ ) and an octreoscan were carried out in search of another digestive localization came back negative. Indicating the primary hepatic location of this tumor. The tumor considered unresectable. Palliative chemotherapy based on etoposide-cisplatin offered to the patient, the latter is decided after having received three injections (Figure 1).
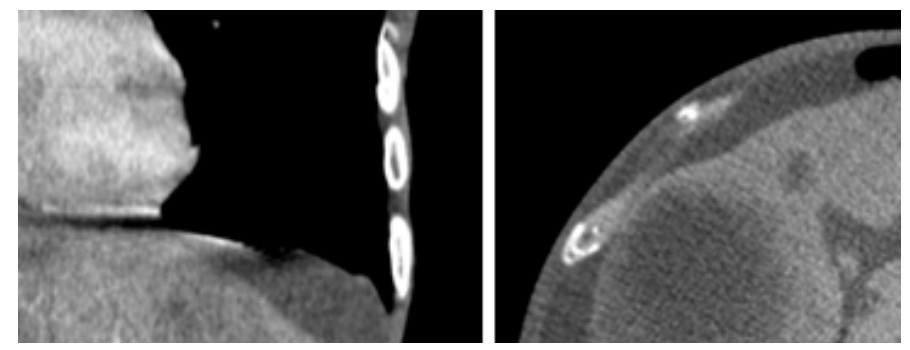

Figure I Liver riddled with nodules, solid, hypodenses, the largest hypovascular.

\section{Discussion}

Neuroendocrinetumors considered rare tumors and little epidemiological data is available. Digestive localizations present approximately $1 \%$ of digestive tumors. ${ }^{3}$ Therefore, the hepatic localization remains even rarer and represents. ${ }^{30} \%$ of all neuroendocrine tumors, ${ }^{1}$ these tumors occur at a relatively youthful age (50years) and they are slightly more frequent in women. ${ }^{4}$ The diagnosis of a primary endocrine tumor of the liver is difficult insofar as the liver is the site of predilection for metastases from extrahepatic endocrine tumors, in particular digestive. The diagnostic process, therefore, comprises two stages: the first is the endocrine diagnosis of the neoplasm. The second is the diagnosis of its primary nature. ${ }^{5}$

Since the first description 60years ago of this rare oncological entity the diagnosis has been a continuum from the preoperative phase for surgical tumors to the postsurgical stage, including long term 
follow up, in search of an extra hepatic primary that is undetectable at the time of diagnosis. The clinical presentation characterized by the non-specificity of the functional and physical signs, sometimes pain or abdominal mass, can be the revealing elements, equally. The case of our patient, while the tumor can be totally asymptomatic and are detected incidentally during an X-ray examination performed for another reason, ${ }^{4}$ or manifested by hormonal syndrome in rare situations ${ }^{4}$ or by Zollinger Elisson syndrome. ${ }^{5}$

Para clinically, the radiological aspects are also nonspecific, in particular the ultrasound can demonstrate a solid hypo or hyperechoic mass, sometimes linked with a cystic component in $18 \%$ of cases, the CT scan The appearance is not equivocal, often with a tumor spontaneously hypodense, associated with a cystic component in $34 \%$ of cases. Contrast uptake in the arterial phase with washout in the portal phase, thus mimicking HCC.6 The diagnostic confirmation is established on the histopathological study of the biopsy fragments, which is not specific and can be confused with that of an HCC. The immunohistochemical study remains the only dependable way to confirm the endocrine nature of the tumor. The specific markers are Chromogranin A, NSE and synaptophysin. ${ }^{6}$ The primary treatment for NETs of the liver remains surgical excision, the extent of which depends on the site and the size of the tumor. When resection is impossible (blubber, multi centric tumor), other therapeutic means can used radiofrequency, selective embolization of the hepatic artery, regional or systemic chemotherapy, and intravenous infusions of somatostatin analogs for symptomatic purposes. ${ }^{6}$

\section{Conclusion}

Primary neuroendocrine tumors of the liver are rare. They are defined as primitive only when extr-hepatic localization, in particular other digestive or extra-digestive organs, IS eliminated by exhaustive insvistigation. This rarity and their nonspecific clinical character means that they are rarely evoked in first intention in front of an isolated hepatic lesion and IT4S mainly on percutaneous biopsy with immunohistochemical study and on octreoscan. The treatment is mainly surgical when it is possible, in the others case, systemic drugs is the other option.

\section{Acknowledgments}

None.

\section{Conflicts of interest}

The authors declare that they have no conflicts of interest to disclose.

\section{Funding}

None.

\section{References}

1. Edmondson HA. Tumors of the Liver and Intrahepatic Bile Ducts. Armed Forces Institute of Pathology. 1958.

2. Quartey B. Primary Hepatic Neuroendocrine Tumor: What Do We Know Now? World J Oncol. 2011;2(5):209-216.

3. Aparicio T, Dominguez-Tinajero S, Mitry E. Digestive and pancreatic endocrine tumors. In: Les cancers digestifs. Paris: Springer-Verlag; 2006. p. 219-240.

4. Lin $\mathrm{CW}$, Lai $\mathrm{CH}$, Hsu CC, et al. Primary hepatic carcinoid tumor: a case report and review of the literature. Cases J. 2009;2:90.

5. Ameur HB, Abdelhedi C, Guermazi F, et al. Tumeur endocrine primitive du foie: un diagnostic difficile. $J$ Afr Hépato-Gastroentérologie. 2012;6:103-106.

6. Lin $\mathrm{CW}$, Lai $\mathrm{CH}$, Hsu CC, et al. Primary hepatic carcinoid tumor: a case report and review of the literature. Cases J. 2009;2(1):90 